\title{
Localized wrinkling of metal films on elastic and liquid substrates
}

\author{
Yi Sun \\ Zhejiang Sci-Tech University, China \\ Norwegian University of Science and Technology, Norway \\ yisun1987@hotmail.com \\ Liping Yan, Benyong Chen \\ Zhejiang Sci-Tech University, China \\ yanliping@zstu.edu.cn,chenby@zstu.edu.cn \\ Wei Song \\ Harbin Institute of Technology, China \\ Norwegian University of Science and Technology, Norway \\ swingways@hotmail.com \\ Filippo Berto \\ Norwegian University of Science and Technology, Norway \\ filippo.berto@ntnu.no
}

\begin{abstract}
Global and localized wrinkling phenomena in films/substrates have been widely studied by means of experiment and theoretical analyses in the past two decades. They have shown broad application prospect in microstructure manufacture. The global wrinkling patterns with well-defined wavelengths can be readily fabricated in the polymer films, the metal films and the surface of modified elastic substrates by $\mathrm{O}_{2}$ plasma or UVO, and they have attracted much attention. For the localized wrinkling patterns, however, most previous studies were limited to polymer films, which while metal films were not properly considered. Recent studies have shown that wrinkles can be localized by constrained edges and cracks in metal films deposited on the elastic and liquid substrates. In this review, we focus on the formation and evolution mechanisms of these localized wrinkle patterns, and we aim to provide some useful guidance for future studies on designing the localized wrinkling patterns with real-time controllable dimension, orientation and morphology in the films/substrates.
\end{abstract}

KEYWORDS. Wrinkles; Cracks; Metal films; Stress; Defects; Constrained edges.

\section{OPEN ACCESS}

Citation: Sun, Y., Yan, L., Chen, B., Song, W., Berto, F., Localized wrinkling of metal films on elastic and liquid substrates, Frattura ed Integrità Strutturale, 48 (2019) 648-665.

Received: 16.12 .2018

Accepted: 22.01 .2019

Published: 01.04.2019

Copyright: (C) 2019 This is an open access article under the terms of the CC-BY 4.0, which permits unrestricted use, distribution, and reproduction in any medium, provided the original author and source are credited. 


\section{INTRODUCTION}

$\mathrm{I}$ $\mathrm{n}$ the past two decades, film/substrate structures have been widely used in many high-tech fields including microelectronic systems, sensors, magnetic recording media, optoelectronic devices and microstructure fabrication. As two different materials, the film and the substrate have different physical and chemical properties. When they are simultaneously subjected to mechanical force, chemical treatment or thermal stress, mismatched deformation between them inevitably occurs. In order to balance the system, some stress modes will form in the films. The stress modes are strongly dependent on the stress properties, the mechanical properties of the films and substrates as well as the interfacial interaction properties between the films and substrates. Under sustained stretching stress, the films will fracture into various crack patterns including straight [1-5], circular [6-8], ridge [9], spiral [6,10-12], radial [7,8,13] and networked cracks [2]. The dimension and morphology of the cracks can be tuned by altering the film thickness and substrate properties. In the case of continuous compressive stress, the films will form various buckling modes such as wrinkles [6,7,14-21], folds [22-24], creases $[23,25]$ and telephone cord buckles $[9,26,27]$. The stress magnitude, the film thickness, the substrate elasticity and the adhesion performance between the films and the substrates can determine the wavelength, amplitude and morphology of the buckling.

For a film/substrate structure, it will form wrinkles when the following four conditions hold: 1) the substrate is thicker than the film, 2) the substrate is softer than the film, 3) the interfacial adhesion is strong and the film tightly adheres to the substrate, 4) the compressive stress of the film is beyond the critical wrinkling stress. In an equi-biaxial stress state, the film tends to spontaneously form labyrinth or herringbone patterns [3,28-30]. Because these wrinkle patterns are irregular and uncontrollable, they are usually considered undesirable results. The ordered wrinkle patterns can be fabricated by using some effective tuning methods to locally alter the equi-biaxial stress state. The stress tuning methods include lithographic pre-patterning of substrate [14,31-33], uniaxial pre-stretch releasing of substrate [34,35], solvent assist [17,36-40], contactline mechanics [41-43], axial compression [44,45], point contact deformation [6,7,16,19,20,22,46-52], simultaneous and sequential release of biaxial pre-stretching [53,54], template adhesion [55-59], constrained edge effect [60,61], selective adhesion to substrate [62], introducing of surface defect [1-4,6-8,36,63], pre-wrinkle directing [64], etc. For example, the homogeneous sinusoidal wrinkle patterns can be fabricated in a stiff film/ elastic substrate by releasing the uniaxial prestrain of the substrate [34]. The localized wrinkling patterns such as radial wrinkles can be fabricated in a polymer film floating on the surface of water by using the method of the point contact deformation [16]. The characteristic parameters can be tuned by controlling the film thickness and stress magnitude. The controllable wrinkles have vast applications in microfluidic channels [34,65], optical gratings [66], flexible electronic devices [67,68], fabricating ordered microstructures [69,70], improving surface adhesion performance [71], culturing viruses [72], measuring film's Young modulus [73,74] and quantifying residual stress [75]. Therefore, the understanding of the formation and evolution mechanisms of homogeneous global and localized wrinkling patterns has become a hot topic for researchers.

Among various film materials, the metal films are widely used in the film/substrate systems to fabricate microstructures due to the relatively simple manufacturing process. For the metal materials such as gold, silver and copper, the chemical and physical properties are relatively stable at high temperature, and the films are generally formed by vacuum evaporation and sputtering deposition. Due to the thermal expansion mismatch between the films and substrates, a high level of residual stress will be stored in the films after deposition. Once the residual stress is beyond the critical wrinkling stress of the films, the global wrinkle patterns will form in the films, which causes the localized wrinkle patterns to be rarely observed in experiments. Previous studies have shown that surface defects such as boundaries, cracks and dusts in the films and the substrates can locally alter equi-biaxial stress state in films and thus alter the local wrinkling morphology [1,3,14]. This implies that the localized wrinkles can be fabricated in the metal films by introducing regularly arranged defects into the films or the substrates. Here, we review some recent progress on the formation and evolution mechanisms of the localized wrinkle patterns in the metal films deposited on the elastic and liquid substrates induced by constrained edges and cracks. The indepth studies of these controllable wrinkle and crack patterns are not only important for understanding the micro-failure mechanics of film structures and devices, but also for further guiding the improvement of the mechanical stability and lifetime of the film structures. This review is outlined as follows. In section 2 , we briefly review the formation mechanisms of the wrinkles and cracks. In section 3, we describe some typically localized wrinkling patterns in the metal films/elastic substrates and their morphological evolution behavior. In section 4, we introduce some localized wrinkling patterns in the metal films/liquid substrates and discuss their evolution mechanisms. Finally, this review is concluded with some suggestions about the future studies of the localized wrinkles. 


\section{FORMATION MECHANISMS OF THE WRINKLES AND CRACKS}

$\mathrm{W}$

hen a metal film is deposited onto a soft substrate, tensile stress and compressive stress are often stored in the film during the deposition and cooling processes, respectively. In order to release the stored stresses, the film and the substrate are usually deformed at the same time. When the deformation is beyond a critical value, the cracks and wrinkles will form in the film to reduce the elastic strain energy of the system. The cracks and wrinkles are widely found in nature and engineering materials. For example, dehydrated fruits and aging skin can cause the formation of wrinkles $[18,76,77]$, and fatigue failure of metal parts can produce progressive cracks [78-91]. Understanding the formation mechanisms of the wrinkles and cracks has a great significance for predicting the material failure.

When the film/substrate is subjected to the compressive stress, the film is placed in an unstable state with high energy. In order to balance the system, the film and the substrate will deform to release the elastic strain energy. Generally, the film has a larger Young's modulus relative to the substrate and is less prone to in-plane deformation. Furthermore, because the film has a small thickness and bending stiffness, it usually generates out-of-plane deformation. When the deformation is beyond a critical value, the wrinkles will be formed to release the compressive stress. Many previous studies have shown that the critical strain $\varepsilon_{c}$ for the outset of wrinkling obeys the following scaling law [14,76,92-94]:

$$
\varepsilon_{c}=-\frac{1}{4}\left(\frac{3 E_{s}\left(1-v_{f}^{2}\right)}{E_{f}\left(1-v_{s}^{2}\right)}\right)^{2 / 3}
$$

In the formula, $E$ is the Young's modulus, $v$ is the Poisson's ratio, the subscripts $f$ and $s$ refer to the film and substrate respectively. The two most important parameters wavelength $\lambda$ and amplitude $A$ for charactering the wrinkles are [14,76,92-95]:

$$
\lambda=2 \pi h\left(\frac{E_{f}\left(1-v_{s}^{2}\right)}{3 E_{s}\left(1-v_{f}^{2}\right)}\right)^{1 / 3} \text { and } A=h\left(\frac{\varepsilon}{\varepsilon_{c}}-1\right)^{1 / 2}
$$

$b$ is the film thickness and $\varepsilon$ is the applied strain.

Eqns. (1) and (2) are classical critical wrinkling condition and characteristic scale of the film under the low deformation regime, respectively. Their application condition is that the stiff film adhered to an isotropically elastic substrate with infinite thickness cannot debond from the substrate. It can find from the Eqn. (1) that the critical wrinkling strain depends on the Young's modulus of the film and substrate. In the case that the substrate is softer and the film is harder, the critical wrinkling strain is smaller, and the film is more prone to form wrinkles. From the Eqn. (2), it is clear that the characteristic wavelength of the wrinkles is dependent on the film thickness and the Young's modulus ratio of the film and the substrate. By energy analysis, Cerda et al. [76] showed that small wavelength will increase the bending energy of the film significantly, large wavelength significantly increase the deformation energy of the substrate, and the competition between the two energies will result in an equilibrium wrinkle wavelength. It can also find that the wrinkle amplitude depends on the film thickness and the applied strain. If the applied strain increases, the amplitude will increase. When the amplitude increases to a critical value, the wrinkles will collapse into the folds [22].

For the metal film, it is difficult to withstand tensile stress because it is quite brittle. According to the Griffith classical criterion, crack will propagate when the elastic energy stored in the film released per unit length overcomes the fracture energy [11],

$$
2 \gamma h e \geq G_{f} h \text { with } e=h \sigma_{t}^{2}\left(1-v_{f}\right) / E_{f}
$$

where $e$ is the elastic energy in the film per unit surface, $\sigma_{t}$ is the tensile stress, $G_{f}$ is the fracture energy per unit area and $\gamma$ is a constant that depends on the mismatch in elastic properties between the film and the substrate. Eqn. (3) shows that the crack propagation depends not only on the tensile stress but also on the film thickness. Below a critical thickness $h=G_{f} E_{f} / 2 \gamma \sigma_{t}^{2}\left(1-v_{f}\right)$ the film is free of cracks $[10,11,96,97]$. Above the critical thickness, the cracks appear. When the 
film thickness increase, the fracture energy will also increase, and it needs a larger tensile stress to break the film. Furthermore, the fracture morphology depends notably on the film thickness, the substrate elastic and the loading conditions (such as temperature, mechanical stretching and loading velocity).

\section{MORPHOLOGIES AND EVOLUTION OF LOCALIZED WRINKLES IN METAL FILM/ELASTIC SUBSTRATE}

$\mathrm{I}$ n 1998, Bowden et al. [14] first reported the microscopic wrinkling in a metal film/elastic substrate, and they observed that the metal film deposited on the flat substrate spontaneously form disordered wrinkles. By prefabricating some regularly arranged patterns on the substrate, they found that the film would form highly ordered wrinkles. Theoretical analysis showed that the patterns on the substrate changed the local compressive stress state and thus altered the local wrinkling morphology. Since then, various global and localized wrinkling patterns have been fabricated in different films/substrates by using some effective stress tuning technologies. The global wrinkles with well-defined wavelengths such as herringbone, sinusoidal and labyrinth patterns can be readily fabricated in various film/substrate materials including the metal films/elastic substrates [2,14,24,28-30], the nonmetal stiff films/elastic substrates [34,95,98-100] and the polymer films/elastic substrates $[33,41-43,101]$. The localized wrinkles such as the radial patterns can be easily formed in the floating polymer films [16,20,22,46,50,51,102] and the surface of modified elastomer [36]. Furthermore, some researchers have used numerical simulations to report the spiral and radial wrinkling patterns in stiff films/elastic substrates [19,52]. However, the localized wrinkling patterns in metal films deposited on the elastic substrates are rarely observed in experiments. It should be emphasized here that these localized wrinkling patterns do not include the buckle-driven delamination and the folds, creases and ridges induced by the post-buckling evolution of wrinkles.

Recent studies show that the wrinkles in the metal films deposited on the elastic substrates can be localized near some surface defect sites. The distribution of the wrinkles is closely related to the formation mechanism of the defects, and the wrinkle morphology is dependent on the defect morphology, the direction of the compressive stress, the film thickness and the elasticity of the substrate. Here, we introduce some localized wrinkle patterns in the metal films deposited on the elastic substrates.

Vandeparre et al. [17] reported some localized wrinkle patterns in Ti film deposited on elastic PS substrate with diffusion of toluene solvent. They found that the wrinkle morphology is closely related to the geometry of the diffusion front. When the solvent diffuses from the polymer edge, a linear diffusion front is yielded, which results in the formation of parallel wrinkles, as shown in Fig. 1A(a). If the solvent diffusion starts from a point-like hole defect at a random site in the film, the wrinkles will develop at the defect site and then expand along radial direction, as shown in Fig. 1A(b). The growth of the wrinkles can be stopped by neighboring patterns, and then these wrinkle patterns will join together, which results in the formation of homogeneous global wrinkle patterns, as shown in Fig. 1B. By changing the rheological behavior of the polymer substrate, they also generated complex hierarchical wrinkled patterns. The experimental result and method present new directions in achieving desired localized wrinkle patterns in metal film deposited on the elastic substrates. Since then, the localized wrinkling of the metal film has begun to attract much attention of some researchers, and some localized wrinkle patterns have been generated in different metal film/substrate structures by introducing regular defects in the metal film or substrate.
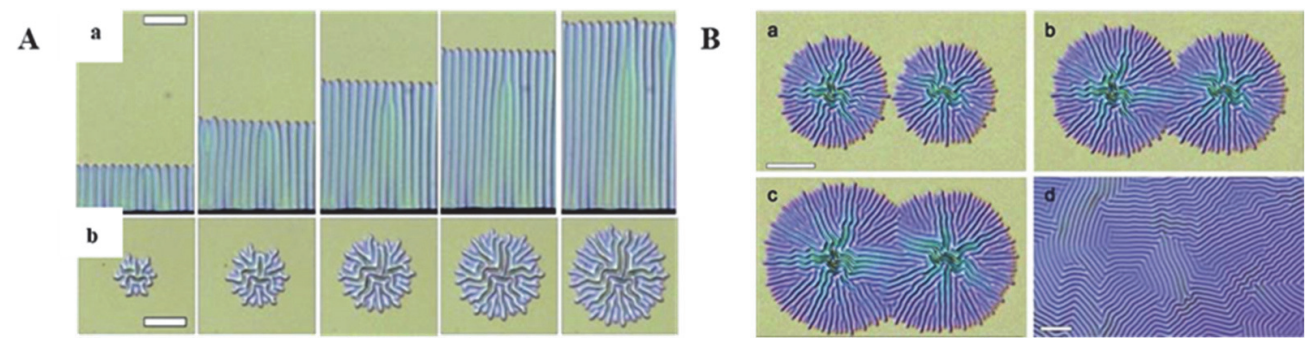

Figure 1: (A) Gradually growing parallel wrinkles (a) and radial wrinkles (b). (B) Successive optical images (a-c) of two radial wrinkles fusing and final image (d) after fusion of several radial wrinkles. The scale bar corresponds to $20 \mu \mathrm{m}$. Images are from ref. [17].

Rand et al. [1] first reported that the surface wrinkles with long-range alignment can be achieved by introducing a simple fracture process in a stiff film. Their experiment results show that the wrinkles are perpendicular to the fracture line and are constrained between the neighboring fracture lines, which implies that the compressive stress near the fracture edge was uniaxial and its direction was parallel to the fracture line. The experimental method provides a possibility to form localized wrinkles by introducing regularly arranged fracture patterns in the metal film or the substrate. Then by effectively controlling 
the evolution of the fracture morphology, it is able to precisely tune the morphological evolution of the localized wrinkles. Recently, Wu et al. [32,63] introduced the curved cracks to the elastic substrates and created some localized wrinkle patterns in a thin Al film deposited on a PDMS substrate by thermal evaporation. They studied the controllable mechanisms of the wrinkle patterns. They observed that the wrinkles unevenly distribute on the convex side of the crack near the irregular cracks, such as shown in Fig. 2A. The ordered wrinkle patterns can be fabricated by patterning the substrate with regular cracks, such as the radial wrinkles induced by concentric circle cracks, as shown in Fig. 2B. Based on theoretical analysis, they found that the compressive stress is always concentrate on the convex side of the curved crack, causing the wrinkles to be localized on the convex side of the crack. On the concave side of the curved crack, however, the compressive stress is released, which results in a suppression effect to the wrinkling. The curvature radius of the curved crack has a significant effect on the distribution of the wrinkles. The smaller curvature radius is more significantly to suppress the wrinkling on the concave side of the curved crack, which can be validated from Fig. 2B. It is clear from Fig. 2B (a) that the radial wrinkles develop from the convex side of $c_{1}$ and then extend to the concave side of $c_{2}$ and disappear. When curvature radius of the curved crack becomes larger, the curvature of the crack cannot afford to suppress the wrinkling at the concave side, thus the wrinkles shown in Fig. 2B (b) run through $c_{2}$ and $c_{3}$. The experimental results provide a new insight into controlling the localized wrinkling in metal film/elastic substrate, and the experimental technique can be developed to effectively control the distribution and orientation of the winkling by introducing the regularly arranged patterns into the substrate before film deposition.

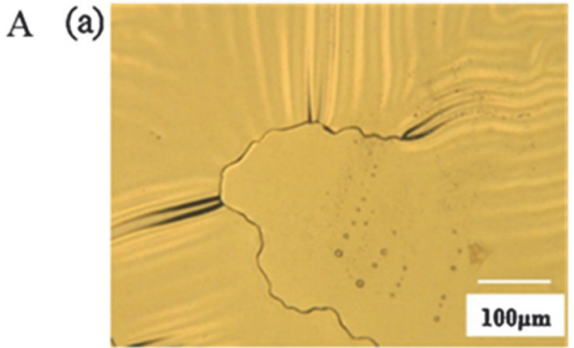

(b)

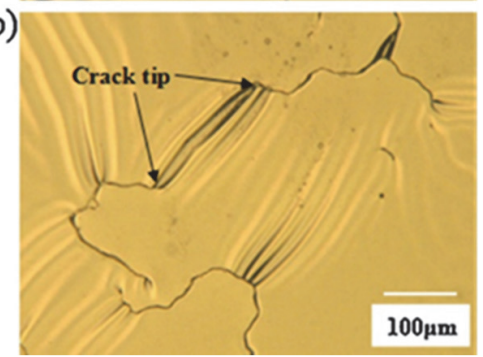

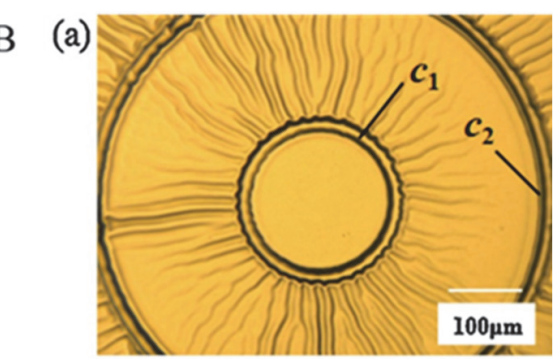

(b)

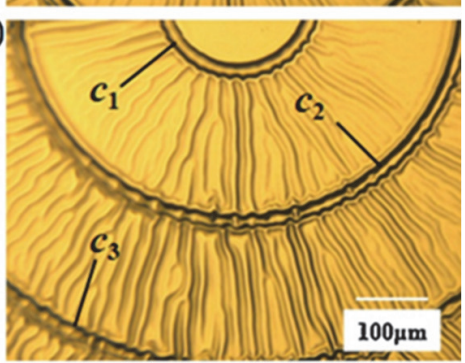

Figure 2: (A) Wrinkle patterns near irregular cracks. (B) Optical image of the radial wrinkles induced by concentric circle cracks $c_{1}, c_{2}$ and c3. Images are from ref. [63].

Many studies show that the deposition of the metal film can slightly increase the temperature of the elastic substrate due to the heat radiation from sputtering source and the bombardment of the metal particles with high energy. During deposition, the substrate is expanded and the metal film is placed under a tensile stress. When the stress reaches a critical value, cracks start to form in the metal film. After deposition, the substrate contracts, and the film is placed under a compressive stress. When the stress is beyond a critical value, disordered wrinkles will appear in the areas away from the cracks. Near the cracks, the metal film undergoes a plastic deformation, and the compressive stress is concentrated in deformation zone, which results in the formation of locally ordered wrinkles. Therefore, by effectively controlling the deposition temperature to produce the fracture patterns, it is able to form the localized wrinkles near the cracks. Recently, Yu et al. [2] studied the formation and evolution of the wrinkled stripes localized by straight crack in a Fe film/PDMS substrate with thermal treatment. The wrinkled stripe is composed of many parallel straight wrinkles normal to the crack plane, and these wrinkles always form on both sides of the new crack, as shown in Fig. 3. They found that the morphologies of the wrinkles and cracks are obviously dependent on the film thickness and the elasticity of the substrate. As the film thickness increases, the crack spacing $s$ decreases and eventually reaches a stable value, the crack number $N$ increases, the old crack width $w$ is almost unchanged while the new crack width increases. Meanwhile, the wrinkle length $L$ decreases, while the wrinkle wavelength $\lambda$ and amplitude $A$ increase significantly, as shown in Fig. 3B. With the increases of the curing time $T_{c}$ of the substrate, the elasticity of the substrate will decrease, which causes the decreases of the crack spacing, the crack width, the 
wrinkled stripe width, the wrinkle wavelength and the wrinkle amplitude, as shown in Fig. 3C. Their analysis shows that the geometrical parameters of the wrinkles and cracks decrease exponentially and eventually reach a stable value. Therefore, the morphologies of the wrinkles and cracks can be effectively tuned by altering the film thickness and the elasticity of the substrate. These experimental results provide a deep understanding for the formation and evolution of localized wrinkle patterns in a metal film deposited on a thermally expanded elastic substrate.

A
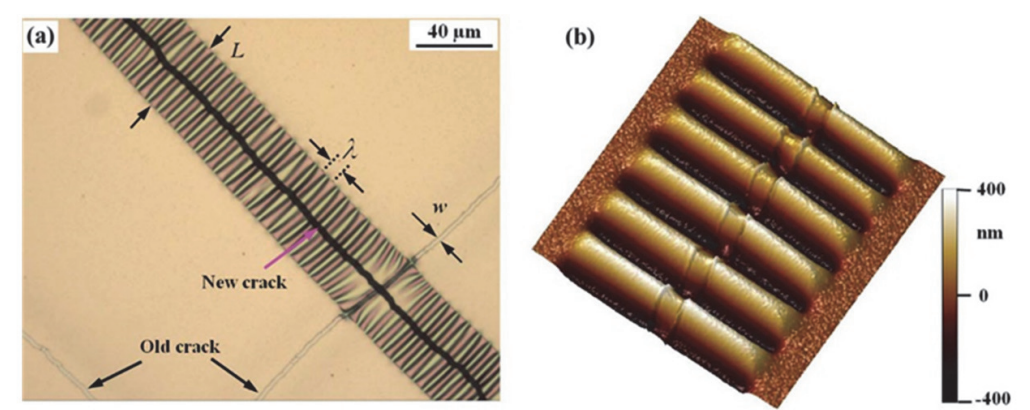

B
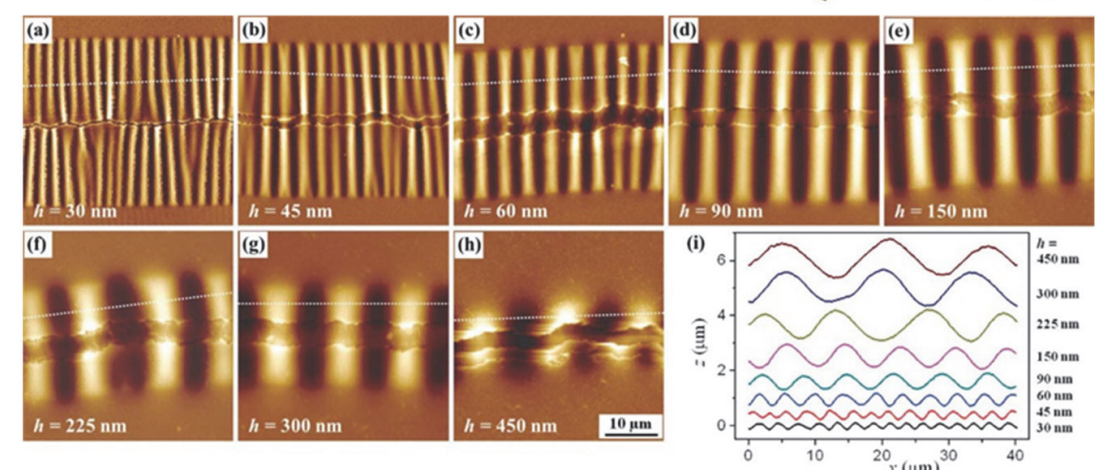

$\mathrm{C}$
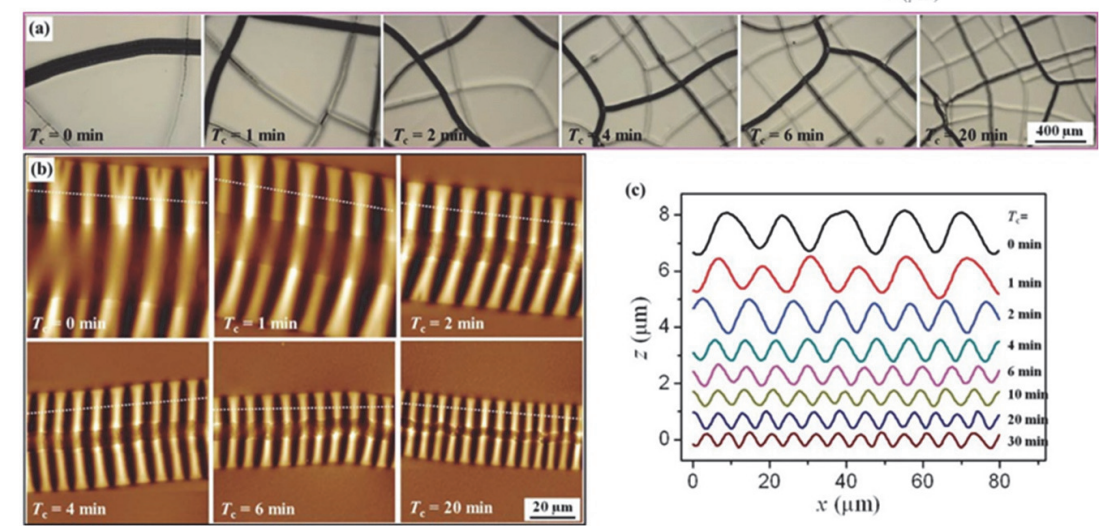

Figure 3: (A) Optical image (a) of the wrinkled stripe and AFM image (b) of the localized wrinkled stripe. (B) Morphological evolution $(\mathrm{a}-\mathrm{h})$ of the wrinkled stripe with film thickness and wrinkle profiles (i) under different film thicknesses. (C) Typical crack morphologies (a) under different curing time, morphological evolution (b) of the wrinkles with curing time and wrinkle profiles (e) under different curing time. Images are from ref. [2].

For the localized wrinkles induced by thermal stress such as reported by $\mathrm{Yu}$ et al. [2], although the morphology of the wrinkles can be tuned by altering the film thickness and the substrate elasticity, the post-buckling evolution of the wrinkles in a given metal film/elastic substrate cannot be tuned in real time. Recently, Sun et al. [6,7] demonstrated a promising prospect for controlling the post-buckling evolution of the localized wrinkles by mechanically inducing the fracture in an initial flat $\mathrm{Au}$ film/PDMS substrate with a micro probe. They found that the morphologies of the wrinkles and cracks are dependent on the loading direction. When an out-of-plane indentation is applied to the Au film/PDMS substrate, the film is induced a compressive stress in the circumferential direction and a tensile in radial direction, which results in the formation of the radial wrinkles and spiral crack near the probe tip [6]. With the increase of the indentation depth, the spiral crack spontaneously propagates along a left-handed or right-handed path, and the propagation of the crack is always accompanied by the wrinkles until the propagation is terminated due to the crack closure, as shown in Fig. 4. The experimental results 
show that the wrinkles are always localized on the concave side of the spiral crack, which are greatly distinguished from those reported by Wu et al. $[32,63]$. On the concave side of the spiral crack, the compressive stress is concentrated, and the wrinkling is more likely to be induced. On the convex side of the spiral crack, however, the compressive stress is relieved since the film outside the crack is no longer constrained spatially through attachment to the rest of the film, which causes the wrinkling to be suppressed. After the spiral crack is closed, the film will form new wrinkles on the convex side of the crack when the compressive stress is beyond the critical wrinkling stress of the film again, then these new wrinkles will experience a secondary bifurcation and evolve into branching patterns, as shown in Fig. 4(b) and (c). The postbuckling evolution of the wrinkles can be tuned by controlling the displacement of the probe. Furthermore, they found that when the tensile stress of the film outside the crack is beyond the breaking strength of the film again, the concentric circle cracks would form in the film.
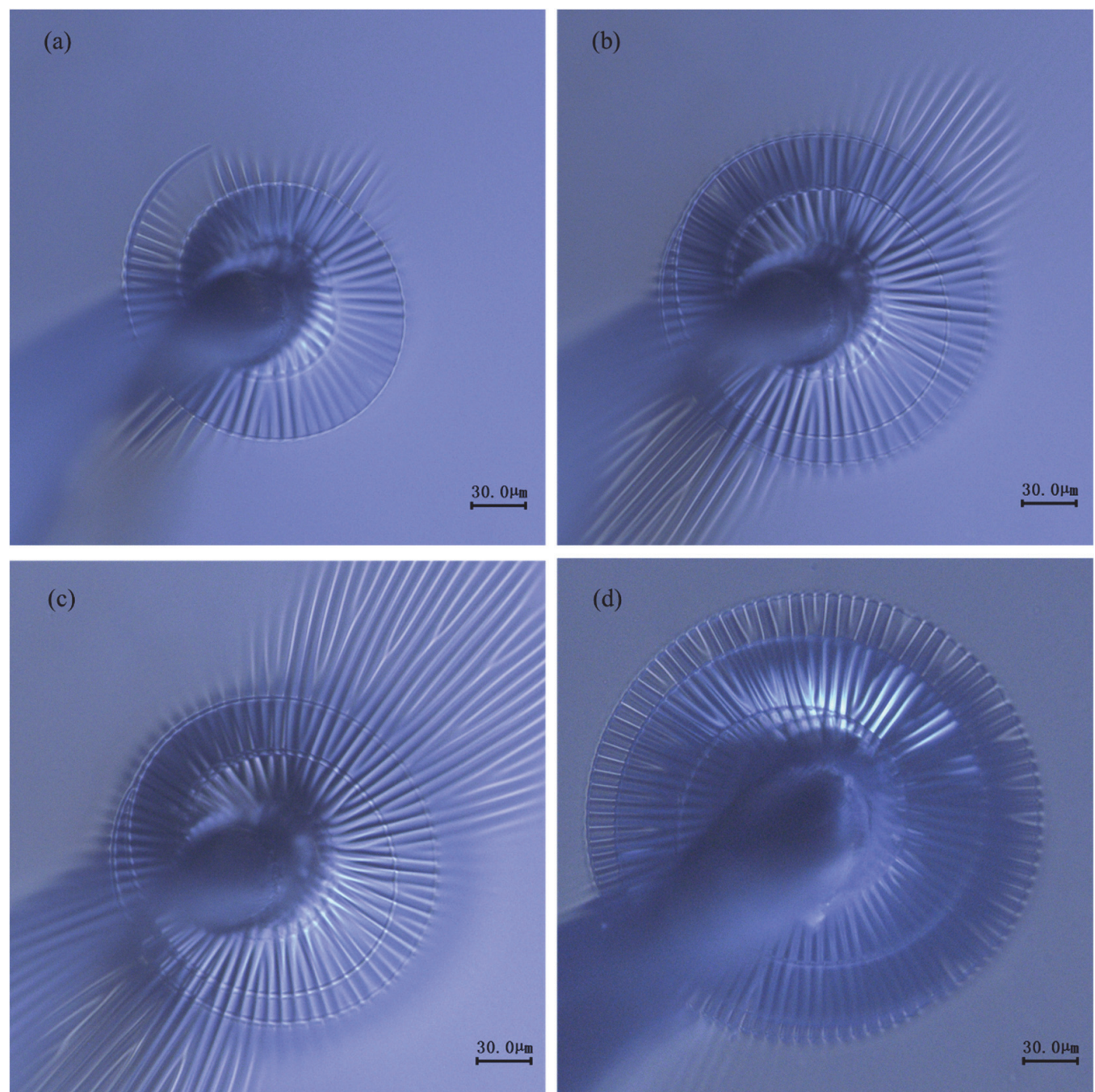

Figure 4: Typical morphologies of the wrinkles and cracks in the Au film/PDMS substrate under out-of-plane indentation, modified from ref. [6].

When an out-of-plane indentation and an in-plane displacement are successively applied to the Au film/PDMS substrate, Sun et al. [7] observed a transition of the localized wrinkles from radial to circumferential, as shown in Fig. 5. Meanwhile, the crack pattern changes from circular to radial. From Fig. 5(a) and (c), it is clear that the the wrinkling always precedes the fracture. Under the out-of-plane indentation, the compressive stress is along the circumferential direction, which results in the formation of the radial wrinkles. They observed that the extended wrinkles tend to split, which causes the local wrinkle wavelength to be maintained in a narrow interval, as shown in Fig. 5(a). With the formation of the crack, the radial wrinkles begin to shrink and are eventually confined within a circular crack, as shown in Fig .5(b). In order to describe the effect of 
the formation of the crack on the local stress of the film, they presented some mathematical models to analyze the evolution of the radial stress $\sigma_{r r}$ and the hoop stress $\sigma_{\theta \theta}$ before and after the crack formation, as described in Eqns. (4) and (5):
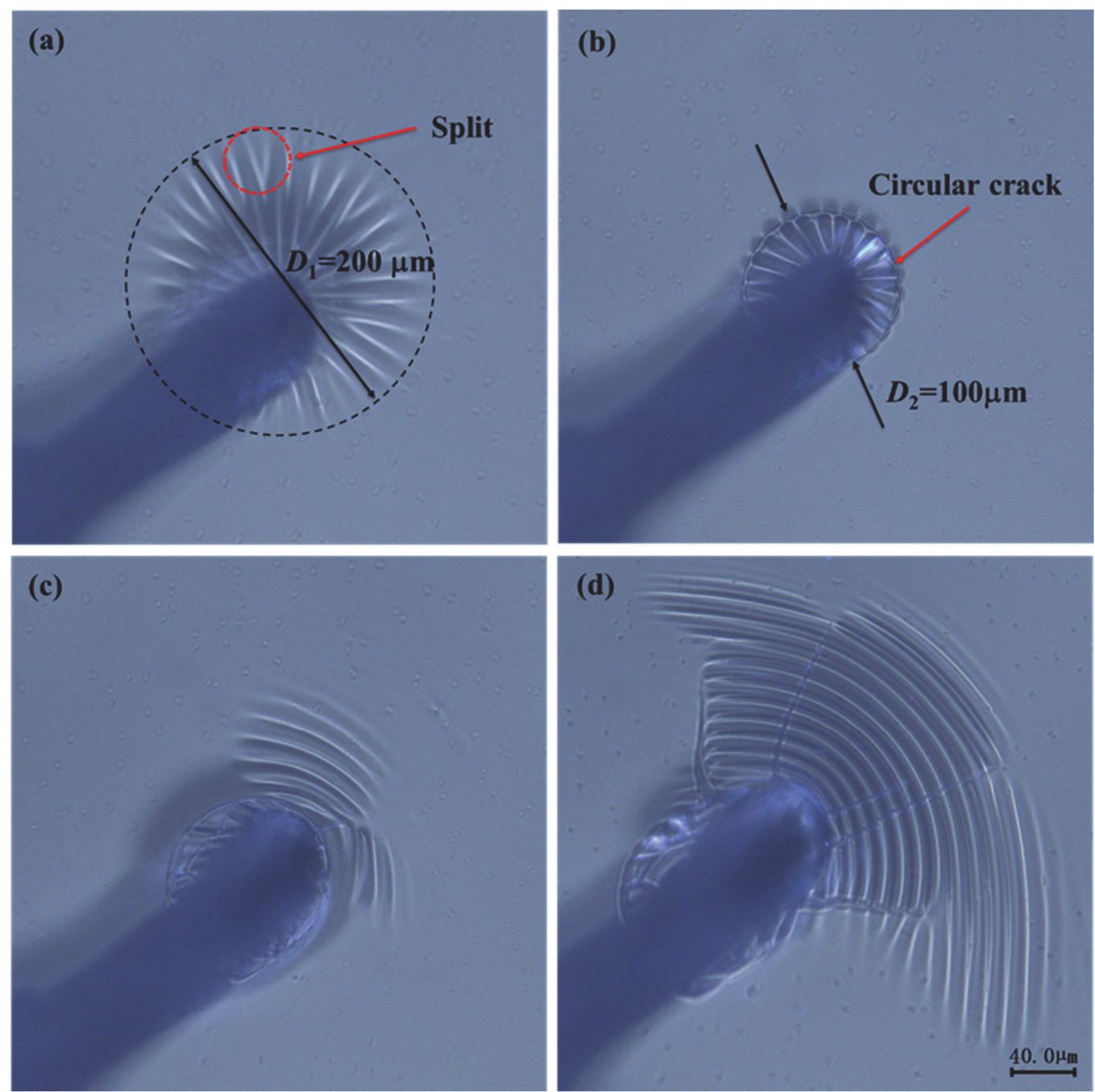

Figure 5: Morphology transition from circular crack with radial wrinkles under out-of-plane indentation to radial cracks with circumferential wrinkles under in-plane displacement, modified from ref. [7].
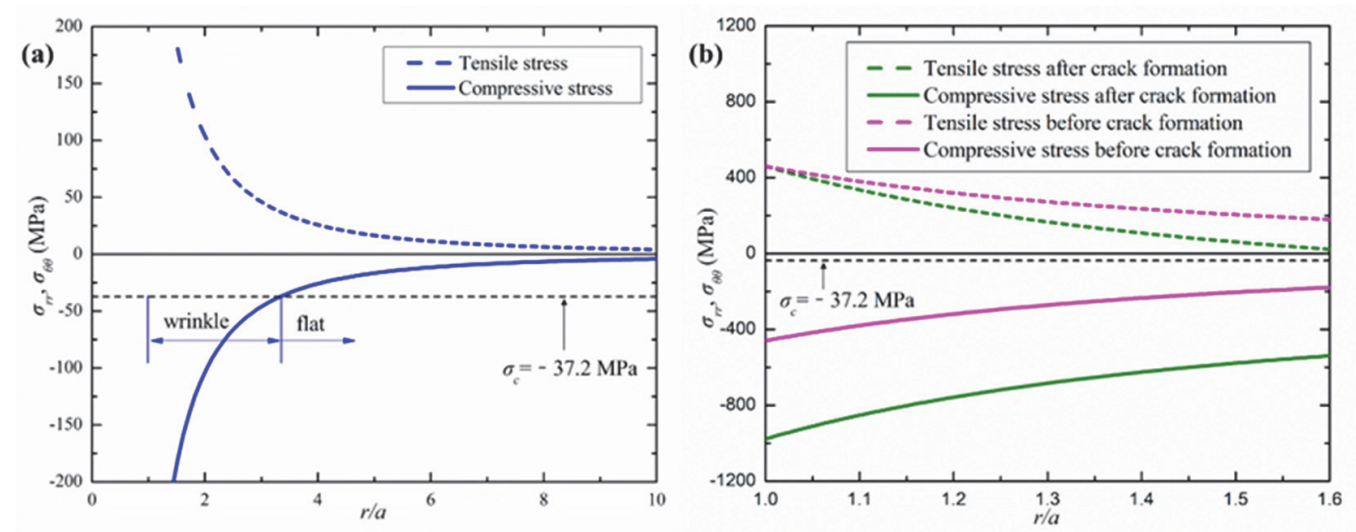

Figure 6: Evolution profiles (a) and (b) of the radial stress $\sigma_{r r}$ (dashed curves) and the hoop stress $\sigma_{\theta \theta}$ (solid curves) with $r / a$ at indentation depths $\omega=18 \mu \mathrm{m}$ (corresponding to Fig. 5(a)) and $\omega=20 \mu \mathrm{m}$ (corresponding to Fig. 5(b)). Modified from ref. [7]. 


$$
\begin{aligned}
& \left\{\begin{array}{l}
\sigma_{r r}=\frac{\tau a^{2}}{r^{2}} \\
\sigma_{\theta \theta}=-\frac{\tau a^{2}}{r^{2}}
\end{array}\right. \\
& \left\{\begin{array}{l}
\sigma_{r r}=\tau\left(\frac{D_{2}^{2}-4 r^{2}}{D_{2}^{2}-4 a^{2}}\right)\left(\frac{a}{r}\right)^{2} \\
\sigma_{\theta \theta}=-\tau\left(\frac{D_{2}^{2}+4 r^{2}}{D_{2}^{2}-4 a^{2}}\right)\left(\frac{a}{r}\right)^{2}
\end{array}\right.
\end{aligned}
$$

Where $\tau$ is the pulling stress at the tip edge, $a$ is the radius of the probe tip, $r$ and $\theta$ axes are along and normal to the radial direction, and $D_{2}$ is the diameter of the circular crack. It is clear that the radial stress $\sigma_{r r}$ is always tensile stress and the hoop stress $\sigma_{\theta \vartheta}$ is always compressive stress. Before the formation of the crack, the tensile stress and the compressive stress have same decrease trend, as shown in Fig. 6(a). After the formation, the tensile stress decrease more sharply and the stress value is zero at the crack edge, while the compressive stress is significantly increased at the crack edge as shown in Fig. 6(b). Therefore, the concave side of the crack can strengthen the wrinkling of the film. After the loading direction changes, the radial stress $\sigma_{r r}$ becomes the compressive stress, and the hoop stress $\sigma_{\theta \theta}$ becomes tensile stress, which results in the formation of the circumferential wrinkles and the radial cracks, as shown in Fig 5(c) and (d). The number of the circumferential wrinkles and radial cracks can be controlled by tuning the displacement of probe.

From the above analysis, the wrinkles in the metal film/elastic substrate can be localized by the surface defects. Mechanically induced curved cracks in the substrate will cause the wrinkles to be distributed on the convex edge of the cracks. However, mechanically induced the curved cracks in the film will cause the wrinkles to be distributed on the concave side of the curved crack. Thermally induced cracks in the film will cause the wrinkles to be distributed on both sides of the cracks. Near the point-like, circular and spiral defects, the compressive stress is along the circumferential direction, which results in the formation of radial wrinkles. In vicinity of the straight cracks, the compressive stress is parallel to the cracks, which leads to the formation of parallel wrinkle stripes. Furthermore, near the radial cracks induced by applying an in-plane displacement to the film/substrate, the compressive stress is radial, which results in the formation of the circumferential wrinkles. Although the wrinkles and cracks in metal films deposited elastic substrates have been widely studied experimentally, their evolution mechanisms and the interaction between them have not been systematically studied in theory, it needs to do further research by theoretical analysis.

\section{MORPHOLOGIES AND EVOLUTION OF LOCALIZED WRINKLES IN METAL FILM/LIQUID SUBSTRATE}

W

ith the in-depth study of the wrinkling of the metal films, the choice of the substrates has been no longer limited to the elastic materials, but has already been expanded to the liquid (viscous) materials $[8,17,28,61,103,104]$. For the metal film/elastic substrate structures, the interfacial adhesion is strong, which generally results in the formation of the homogeneous wrinkle patterns. For the metal film/liquid substrate structures, the interfacial adhesion is relatively weak, recent studies show that they usually form hierarchical wrinkle patterns near the constrained edges and some defect sites when the thickness of the substrates is much larger than that of the films.

Vandeparre et al. [17] first reported the hierarchical wrinkle patterns in Ti film deposited on viscous PS substrate by solvent diffusion, as shown in Fig. 7. They found that the diffusion of the solvent changed the mechanical properties of the polymer substrate, and the wrinkle morphology is dependent on the rheological properties of the substrate and the geometry of the diffusion front. As the distance from the diffusion front and the diffusion time increase, the wrinkle wavelength increases accordingly, as shown in Fig. 7(a). The substrate with lower $M_{w}$ (molecular weight) will induce the wrinkles with larger wavelength, as shown in $7(\mathrm{~b})\left(M_{w}=15 \mathrm{KDa}\right)$ and $(\mathrm{c})\left(M_{w}=75 \mathrm{KDa}\right)$. After theoretical analysis, they got the evolution relationship of the hierarchical wrinkle patterns:

$$
\lambda \sim\left(h H_{p}\right)^{1 / 2}\left(E_{f} / \eta\right)^{1 / 6} t^{1 / 6}
$$


Here, $H_{p}$ is the thickness of the substrate, $\eta$ is the substrate viscosity and $t$ is the time. Their analysis suggests that the substrate at the diffusion front will become soft with diffusion of the solvent, causing the film to wrinkle. Near the edges and defects, the substrate produces a purely viscous behavior due to being plasticized for a longer period of time, which results in the formation of the wrinkles with large wavelength. Yin and Ni et al. [105,106] conducted an in-depth study of the mechanism of the hierarchical wrinkle patterns, and they found that the modulus gradient of the film or substrate could result in the formation of the hierarchical wrinkle patterns. Ni et al. [106] gave the relationship between the total elastic energy $U$ per unit area in the wrinkled state and the wrinkle wavelength $\lambda$ :

$$
\frac{U}{U_{0}}=1-\left[1-\frac{1}{\varepsilon_{p r e}}\left(\frac{\pi^{2} b^{2}}{3 \lambda^{2}}+\frac{\lambda \bar{E}_{s}}{4 \pi b \bar{E}_{f}}\right)\right]^{2}
$$

where $U_{0}=b \bar{E}_{f} \varepsilon_{p r e}^{2} / 2, \bar{E}_{f}=E_{f} /\left(1-v_{f}^{2}\right), \bar{E}_{s}=E_{s} /\left(1-v_{s}^{2}\right)$, and $\varepsilon_{p r e}$ is the pre-strain. Eqn. (7) shows that if the modulus ratio of the film and the substrate is a constant, the wrinkles have a uniform and stable wavelength. If the modulus ratio changes with position, the total elastic energy will change, indicating that the wrinkles may have different wavelengths. This theory can well explain the formation of the hierarchical wrinkle patterns reported by Vandeparre et al. [17].

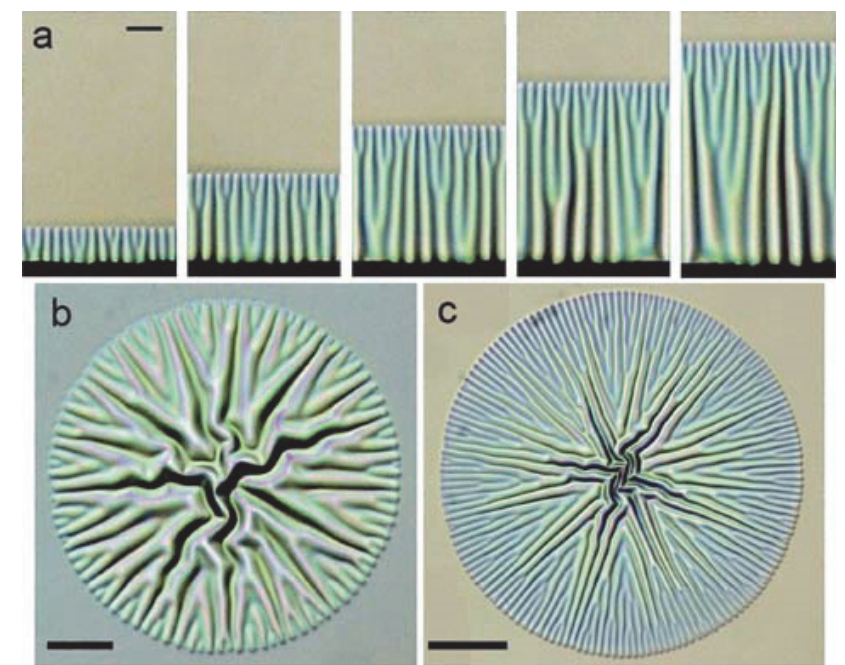

Figure 7: (a) Successive optical images of the growth of the hierarchical wrinkles near the film edge. (b) and (c) Typically hierarchical wrinkle patterns near the hole defects in the films deposited on the substrates with different molecular weight. The scale bar corresponds to $10 \mu \mathrm{m}$. Images are from ref. [17].

Vandeparre et al's experimental results [17] show that the wavelength and amplitude of the hierarchical wrinkles are the largest at the film edges and defect sites. For the metal film deposited on the liquid substrate without constrained edges, the further diffusion of the solvent molecules will cause the localized wrinkles to evolve into a global pattern eventually. When a thin metal film deposited on a thermally expanded liquid substrate with constrained edges, what about the morphology and evolution of the wrinkles? In recent years, some researchers have carried out a series of research works in this area. The following is a brief review of the progress in this area.

$\mathrm{Yu}$ et al. [61] reported the wrinkling in the $\mathrm{Cr}$ film deposited on silicone oil drop. They found that the constrained edges limit the developments of the wrinkle wavelength and amplitude and result in the formation of the hierarchical wrinkle patterns. They show that the formation and morphology of the wrinkles are strongly dependent on the film thickness and the size of the silicone oil drop. For the film with smaller thickness, the wrinkles only form at the inner edge of the silicone oil drop, as shown in Fig. 8A(a). As the film thickness increases, the straight wrinkles decrease gradually, and the hierarchical wrinkle patterns begin to form, as shown in Fig. $8 \mathrm{~A}(\mathrm{~b})$. When the film thickness reaches a critical value, the hierarchical wrinkles will spread all over the oil drop. With the further increase of the film thickness, the wrinkles begin to decrease from the edge of the oil drop, as shown in Fig. 8A(c). Furthermore, they observed that if the size of the silicone oil drop is quite small, no wrinkles form in the film. When the diameter of the oil drop increases to a critical value, the wrinkles first appear in the center of the oil drop and then grow outward until they spread all over the oil drop, as shown in Fig. 8B. The wrinkle 
wavelength is only dependent on the distance starting from the oil drop edge and increases with the distance. The wrinkle amplitude not only relates to the distance, but also depends on the film thickness and the oil drop size. The experimental results show that the wrinkle amplitude increases with the distance and the oil drop size but decreases with the film thickness.

A
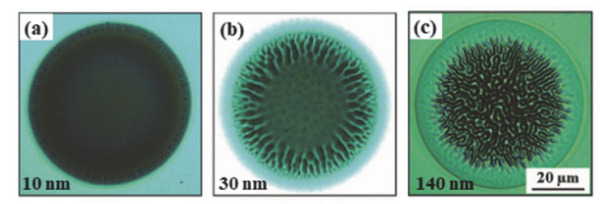

B

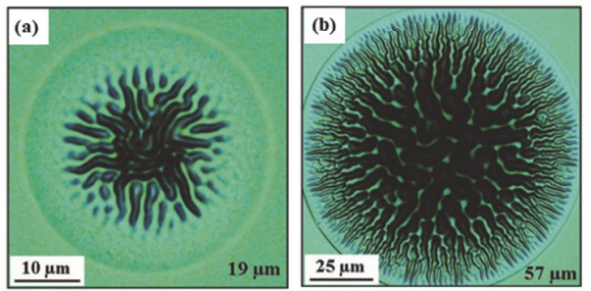

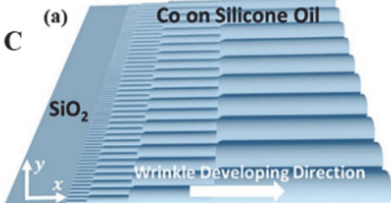

(c)

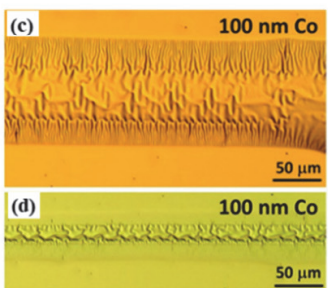

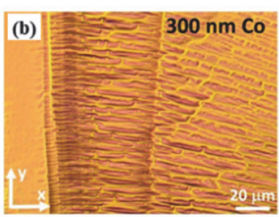

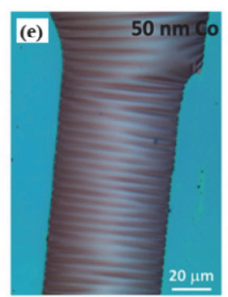

Figure 8: (A) Wrinkle morphology at different film thicknesses [61]. (B) Wrinkle morphology at different diameters of the silicone oil drops [61]. (C) Schematic (a) of hierarchical wrinkles, optical micrograph (b) of self-similar hierarchical wrinkles, and optical micrographs (c-e) of hierarchical wrinkles on cobalt films with various thicknesses deposited on different silicone oil lines [104].

Deng et al. [104] described the formation mechanism of hierarchical wrinklons in a thin metal film (cobalt/chromium) deposited on a silicone oil meniscus. They observed that the hierarchical and self-similar wrinkle patterns initially develop at the silicone oil edge, and grow toward the center of the oil, as shown in Fig. 8C(b-e). They showed that these wrinkles can produce up to 5 hierarchical wrinklons transitions near the constrained edges, and they exhibit a constant wavelength over a distance before transitioning to the next hierarchical wavelength, as depicted in Fig. 8C(a). The hierarchical transitions in wrinkle wavelength follows a relationship: $\lambda_{n+1}=2 \lambda_{n}=2^{n-1} \lambda_{1}(n \geq 1)$, where $n$ is number of the hierarchical transitions.

These transitions will stop when the wrinkle wavelength reaches the bulk wavelength: $\lambda_{n+1} \geq \lambda_{\text {Bulk }}$. The hierarchical transition number and region are dependent on the film thickness and the size of the silicone oil. The thinner films lead to fewer transitions, and smaller silicone oil droplets or lines lead to less transition regions. Based on scaling analysis and energy balance, they got a linear relation between the length and the amplitude of the wrinkles: $L(\lambda) \sim A(\lambda)$. The experimental results and analyses provide theoretical support for the fabrication of hierarchical wrinkles in the metal film/liquid substrate. It is well known that the deposition of the film can change the mechanical properties of the top surface of the substrate due to the bombardment of the high energy particles, creating a thin modified layer between the substrate and the film. For the elastic substrate, the top surface can be modified to form a rigid layer. For the liquid substrate, the top surface can be polymerized to form an elastic layer. When the substrate is very thick, effect of the modified layer on the wrinkle morphology can be neglected. If the thickness of the substrate is finite and even reaches several hundred nanometers, the metal particles may penetrate the substrate, and the substrate may be completely modified with the increase of the deposition time, thus the effect of the modified layer on the wrinkle morphology cannot be neglected. Recently, Yu et al. [103] and Zhang et al. [8] studied the wrinkling in the Fe film deposited on the silicone oil droplet with finite thickness. Yu et al. [103] found that when the silicone oil drop is very small, the film tends to form dotted or short linear shape wrinkles, as shown in Fig. 9(a) and (b). With the increase of the diameter of the oil drop, the linear wrinkles gradually evolve into labyrinth patterns in the center region of the oil drop, as shown in Fig. $9(\mathrm{c}-\mathrm{g})$. When the diameter of the oil drop is beyond a critical value, cracks begin to form in the film, as shown in 9(h). Zhang et al. [8] found that the drop diameter $d$ has prominent influence on the crack morphology. In a small diameter range, the Fe film tends to form a radial cracks across the drop interior or center, as shown in Fig. 10(a) and (b), while the film tends to form circle cracks along the drop edge in a large diameter range, as shown in Fig. 10(c) and (d). Near the radial cracks, the straight wrinkles with the uniform wavelength and amplitude form on both sides of the cracks. In the vicinity of the circle cracks, however, the wrinkle wavelength and amplitude are quite different on both sides of the cracks. The wavelength and amplitude of the wrinkles inside the circle cracks are obviously larger than those outside of the circle cracks. These experimental results provide a deep insight into the wrinkling and fracture of the metal films deposited on the liquid substrate with constrained edges and the complex interaction between the wrinkles and cracks. 

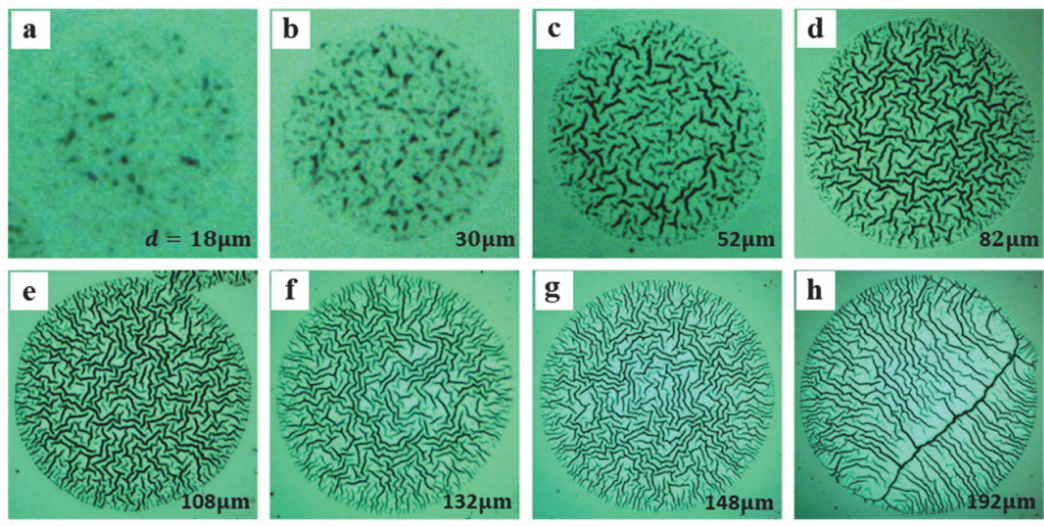

Figure 9: Morphological evolution of the wrinkles with the diameter of silicone oil drop [103]. The data appeared in the bottom-right corners of each image represents the diameter of the silicone oil droplet.
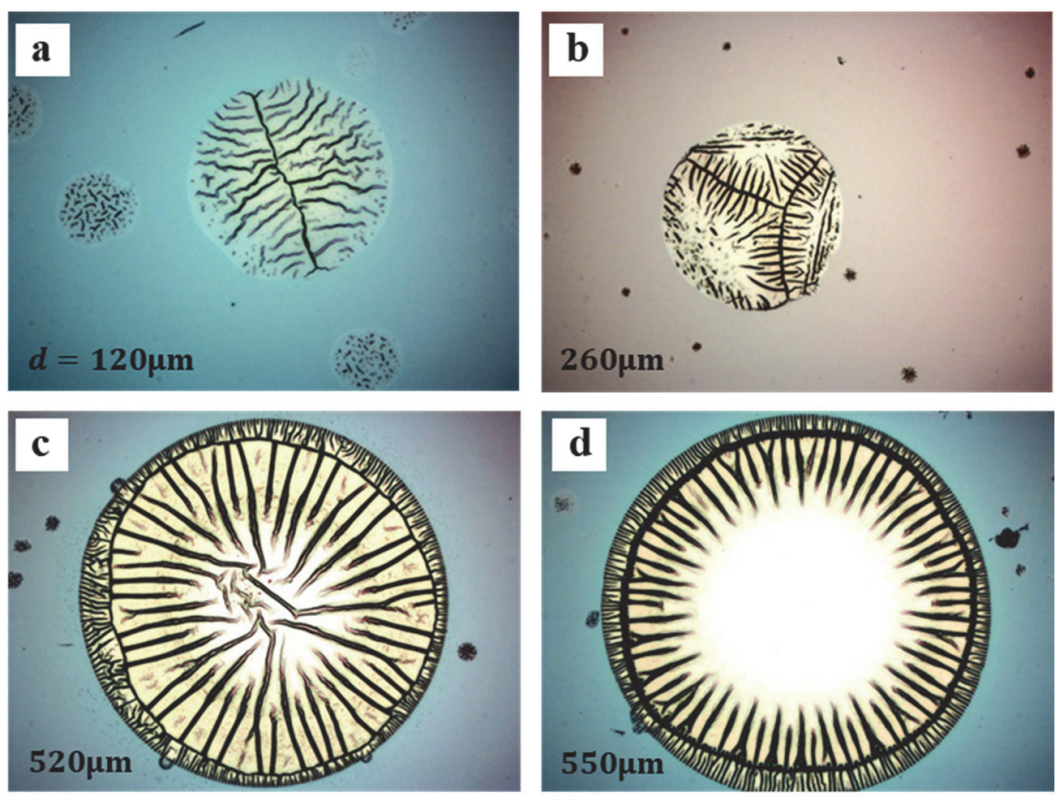

Figure 10: Typical morphologies of the wrinkles and cracks under different diameters of silicone oil drops [8]. The value appeared in the lower-left part of each image represents the diameter of the silicone oil droplet.

It can be seen from the above analysis that the wrinkles in the metal film/liquid substrate can be localized by the constrained edges and the surface defects. The wrinkle morphology is closely related to the edge shape, the defect morphology, the film thickness and the size of the substrate. When the metal film is deposited on an infinitely thick substrate, it tends to form hierarchical wrinkle patterns. When the metal film is deposited on a finitely thick substrate, it tends to form disordered wrinkles, and ordered wrinkle patterns can be observed near the constrained edges and surface defects. Because the study on the wrinkling of the metal films deposited on liquid substrates developed only in recent years, and the experiment and theory are not perfect enough, it needs to be made further investigation.

\section{CONCLUSIONS AND PROSPECTS}

$\mathrm{T}$

his review summarizes some recent study advances of the localized wrinkles in metal films deposited on elastic and liquid substrates, including the formation mechanisms, the ways to control different patterns, and their morphological evolution, which may help to deepen the understanding of the formation and evolution mechanisms for some localized wrinkling phenomena in nature and engineering materials. It is well known that the homogeneous global wrinkle patterns have been widely used in many high-tech applications. For the localized wrinkle patterns, however, many possible applications are limited due to restriction from the length scale. In order to use the localized wrinkle patterns as 
functional devices, it requires further experimental and theoretical researches about how to generate the localized wrinkles with real-time controllable dimension, orientation and morphology. These further studies include designing the defect array in the films or substrates, controlling density and morphology of the defects, adjusting the magnitude and direction of the compressive stress in the films, and tuning the adhesion property between the films and the substrates, etc.

With the in-depth study of the wrinkles in the film/substrate structures, the post-buckling patterns of the local wrinkles have attracted much attention. For example, experiment study shows that the localized wrinkles in a floating polymer film will collapse into folds when the wrinkle amplitude increase with applied strain, and the energy of single fold formation nearly linearly the film thickness [22]. Theoretical analysis demonstrates that the localized wrinkles in a stiff film/compliant substrate may experience a secondly bifurcation and evolve into branching patterns with the increase of the local pre-stretch, and the critical wrinkling strain and the wrinkle number are closely related to the geometric and mechanical parameters of the system [52]. There is a deeper understanding of how the mechanical properties (pre-strain, modulus ratio), geometric parameters (thickness ratio) and interfacial adhesion properties of the film and substrate lead to more complex buckling modes. However, the in-situ monitoring of the post-buckling evolution of the localized wrinkles has not been studied systematically in experiment and theory. In the future, we need to develop new experimental techniques and theoretical analysis methods to finely characterize the dynamic evolution of the post-buckling morphology of the wrinkles.

The real-time controllable evolution of the localized wrinkle morphology and its in-situ monitoring can help us understand the dynamic growth processes of the wrinkled morphologies of some cells and tissues such as bacterial cell, human neutrophil cell, brain cortex, small intestine and tumor tissue [18,107-111]. For example, Tallinen et al. [111] mimicked the growth of the human brain cortex by using solvents to swell the outer layers of a 3D-printed layered gel-brain to yield surface folding patterns, vividly reproducing the formation process of the sulci and gyri of the brain. We expect that the localized wrinkle patterns with controllable morphology will play an increasingly important role in the applications of some engineering materials and biological systems.

\section{REFERENCES}

[1] Rand, C.J., Sweeney, R., Morrissey, M., Hazel, L., Crosby, A.J. (2008). Fracture-induced alignment of surface wrinkles, Soft Matter, 4(9), pp. 1805-1807, DOI: 10.1039/b802271b.

[2] Yu, S., Zhang, X., Xiao, X., Zhou, H., Chen, M. (2015). Wrinkled stripes localized by cracks in metal films deposited on soft substrates, Soft Matter 11(11), pp. 2203-2212, DOI: 10.1039/c5sm00105f.

[3] Sun, Y., Yan, L., Li, C., Chen, B. (2018). Evolution of local wrinkles near defects on stiff film/elastic substrate, Eur. Phys. J. E, 41(3), p. 31, DOI: 10.1140/epje/i2018-11637-4

[4] Gao, T.X., Sun, Y.D., Feng, Y.F., Yu, S.J. (2016). Morphological evolutions of iron films on PDMS substrates under uniaxial compression/tension, Philos. Mag., 96(28), pp. 2943-2952, DOI: 10.1080/14786435.2016.1219457.

[5] Wu, K., Zhang, J.Y., Li, J., Wang, Y.Q., Liu, G., Sun, J. (2015). Length-scale-dependent cracking and buckling behaviors of nanostructured $\mathrm{Cu} / \mathrm{Cr}$ multilayer films on compliant substrates, Acta Mater., 100, pp. 344-358, DOI: 10.1016/j.actamat.2015.08.055.

[6] Sun, Y., Yan, L., Li, C., Chen, B. (2018). Evolution of wrinkles with spiral crack on stiff film/compliant substrate under controllable micro-probe loading, Appl. Surf. Sci., 455, pp. 37-44, DOI: 10.1016/j.apsusc.2018.05.079.

[7] Sun, Y., Yan, L., Chen, B. (2018). Arcuate wrinkling on stiff film/compliant substrate induced by thrust force with a controllable micro-probe, Eur. Phys. J. E, 41(8), p. 89, DOI: 10.1140/epje/i2018-11700-2.

[8] Zhang, Y.J., Yu, S.J., Zhou, H., Cai, P.G. (2018). Fracture and wrinkle patterns in metal films deposited on liquid meniscuses, Surf. Rev. Lett., 25(04), p. 1850088, DOI: 10.1142/S0218625X18500889.

[9] Yu, S.J., Li, S.C., Ni, Y., Zhou, H. (2017). Size dependent morphologies of brittle silicon nitride thin films with combined buckling and cracking, Acta Mater., 127, pp. 220-229, DOI: 10.1016/j.actamat.2017.01.038.

[10] Lazarus, V., Pauchard, L. (2011). From craquelures to spiral crack patterns: Influence of layer thickness on the crack patterns induced by desiccation, Soft Matter, 7(6), pp. 2552-2559, DOI: 10.1039/c0sm00900h.

[11] Marthelot, J., Roman, B., Bico, J., Teisseire, J., Dalmas, D., Melo, F. (2014). Self-replicating cracks: A collaborative fracture mode in thin films, Phys. Rev. Lett., 113(8), p. 085502, DOI: 10.1103/PhysRevLett.113.085502.

[12] Matsuda, F., Sobajima, T., Irie, S., Sasaki, T. (2016). Spiral crack patterns observed for melt-grown spherulites of poly(Llactic acid) upon quenching, Eur. Phys. J. E, 39(4), p. 41, DOI: 10.1140/epje/i2016-16041-6.

[13] Zhang, Y., Qian, Y., Liu, Z., Li, Z., Zang, D. (2014). Surface wrinkling and cracking dynamics in the drying of colloidal droplets, Eur. Phys. J. E, 37(9), p. 84, DOI: 10.1140/epje/i2014-14084-3. 
[14] Bowden, N., Brittain, S., Evans, A.G., Hutchinson, J.W., Whitesides, G.M. (1998). Spontaneous formation of ordered structures in thin films of metals supported on an elastomeric polymer, Nature, 393(6681), pp. 146-149, DOI: $10.1038 / 30193$.

[15] Li, C., Zhang, X., Cao, Z. (2005). Materials science: Triangular and Fibonacci number patterns driven by stress on core/shell microstructures, Science, 309(5736), pp. 909-911, DOI: 10.1126/science.1113412.

[16] Huang, J., Juszkiewicz, M., De Jeu, W.H., Cerda, E., Emrick, T., Menon, N., Russell, T.P. (2007). Capillary wrinkling of floating thin polymer films, Science, 317(5838), pp. 650-653, DOI: 10.1126/science.1144616.

[17] Vandeparre, H., Gabriele, S., Brau, F., Gay, C., Parker, K.K., Damman, P. (2010). Hierarchical wrinkling patterns, Soft Matter, 6(22), pp. 5751-5756, DOI: 10.1039/c0sm00394h.

[18] Chen, X., Yin, J. (2010). Buckling patterns of thin films on curved compliant substrates with applications to morphogenesis and three-dimensional micro-fabrication, Soft Matter, 6(22), pp. 5667-5680, DOI: $10.1039 / \mathrm{c} 0 \mathrm{sm} 00401 \mathrm{~d}$.

[19] Wang, J.W., Cao, Y.P., Feng, X.Q. (2014). Archimedean spiral wrinkles on a film-substrate system induced by torsion, Appl. Phys. Lett., 104(3), p. 031910, DOI: 10.1063/1.4862922.

[20] Vella, D., Huang, J., Menon, N., Russell, T.P., Davidovitch, B. (2015). Indentation of ultrathin elastic films and the emergence of asymptotic isometry, Phys. Rev. Lett., 114(1), p. 014301, DOI: 10.1103/PhysRevLett.114.014301.

[21] Becton, M., Wang, X. (2016). Tailoring patterns of graphene wrinkles by circular torsion, Appl. Surf. Sci., 363, pp. 1320, DOI: $10.1016 /$ j.apsusc.2015.11.091.

[22] Holmes, D.P., Crosby, A.J. (2010). Draping films: A wrinkle to fold transition, Phys. Rev. Lett., 105(3), p. 038303, DOI: 10.1103/PhysRevLett.105.038303.

[23] Al-Rashed, R., López Jiménez, F., Marthelot, J., Reis, P.M. (2017). Buckling patterns in biaxially pre-stretched bilayer shells: Wrinkles, creases, folds and fracture-like ridges, Soft Matter, 13(43), pp. 7969-7978, DOI: 10.1039/c7sm01828b.

[24] Yu, S., Sun, Y., Li, S., Ni, Y. (2018). Harnessing fold-to-wrinkle transition and hierarchical wrinkling on soft material surfaces by regulating substrate stiffness and sputtering flux, Soft Matter, 14(32), pp. 6745-6755, DOI: $10.1039 / \mathrm{c} 8 \mathrm{sm} 01287 \mathrm{c}$.

[25] Lestringant, C., Maurini, C., Lazarus, A., Audoly, B. (2017). Buckling of an elastic ridge: Competition between wrinkles and creases, Phys. Rev. Lett., 118(16), p. 165501, DOI: 10.1103/PhysRevLett.118.165501.

[26] Yu, S.J., Xiao, X.F., Chen, M.G., Zhou, H., Chen, J., Si, P.Z., Jiao, Z.W. (2014). Morphological selections and dynamical

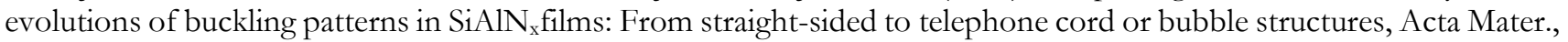
64, pp. 41-53, DOI: 10.1016/j.actamat.2013.11.038.

[27] Li, S.C., Yu, S.J., He, L., Ni, Y. (2018). Effects of ridge cracking and interface sliding on morphological symmetry breaking in straight-sided blisters, J. Mech. Phys. Solids, 112, pp. 637-649, DOI: 10.1016/j.jmps.2017.10.001.

[28] Yu, S.J., Du, Y.P., Sun, Y.D., Ye, Q.L., Zhou, H. (2017). Wrinkling patterns in metal films sputter deposited on viscoelastic substrates, Thin Solid Films, 638, pp. 230-235, DOI: 10.1016/j.tsf.2017.07.051.

[29] Li, K., Wang, J., Shao, B., Xiao, J., Zhou, H., Yu, S. (2018). Wrinkling patterns of tantalum films on modulus-gradient compliant substrates, Thin Solid Films, 654, pp. 100-106, DOI: 10.1016/j.tsf.2018.03.094.

[30] Yu, S., Sun, Y., Du, Y., Zhou, H. (2018). Harnessing surface wrinkling in film-substrate system by precisely controlling substrate modulus, Thin Solid Films, 660, pp. 353-357, DOI: 10.1016/j.tsf.2018.06.042.

[31] Ebata, Y., Crosby, A.J. (2014). Wrinkling membranes with compliant boundaries, Soft Matter, 10(12), pp. 1963-1968, DOI: $10.1039 / \mathrm{c} 3 \mathrm{sm} 52750 \mathrm{f}$.

[32] Wu, D., Yin, Y., Xie, H., Shang, Y., Li, C., Wu, L., Dai, X. (2014). Controlling the surface buckling wrinkles by patterning the material system of hard-nano-film/soft-matter-substrate, Sci. China Physics, Mech. Astron., 57(4), pp. 637-643, DOI: $10.1007 / \mathrm{s} 11433-014-5391-7$.

[33] Wang, R., Long, Y., Zhu, T., Guo, J., Cai, C., Zhao, N., Xu, J. (2017). Fabrication of oriented wrinkles on polydopamine/polystyrene bilayer films, J. Colloid Interface Sci., 498, pp. 123-127, DOI: 10.1016/j.jcis.2017.03.035.

[34] Khare, K., Zhou, J., Yang, S. (2009). Tunable open-channel microfluidics on soft poly(dimethylsiloxane) (PDMS) substrates with sinusoidal grooves, Langmuir, 25(21), pp. 12794-12799, DOI: 10.1021/la901736n.

[35] Zhang, S., Zhan, Q., Yu, Y., Liu, L., Li, H., Yang, H., Xie, Y., Wang, B., Xie, S., Li, R.W. (2016). Surface morphology and magnetic property of wrinkled FeGa thin films fabricated on elastic polydimethylsiloxane, Appl. Phys. Lett., 108(10), p. 102409, DOI: $10.1063 / 1.4943943$.

[36] Chung, J.Y., Nolte, A.J., Stafford, C.M. (2009). Diffusion-controlled, self-organized growth of symmetric wrinkling patterns, Adv. Mater., 21(13), pp. 1358-1362, DOI: 10.1002/adma.200803209.

[37] Breid, D., Crosby, A.J. (2011). Effect of stress state on wrinkle morphology, Soft Matter, 7(9), pp. 4490-4496, DOI: $10.1039 / \mathrm{c} 1 \mathrm{sm} 05152 \mathrm{k}$. 
[38] Lucantonio, A., Roché, M., Nardinocchi, P., Stone, H.A. (2014). Buckling dynamics of a solvent-stimulated stretched elastomeric sheet, Soft Matter, 10(16), pp. 2800-2804, DOI: 10.1039/c3sm52941j.

[39] Stenberg, H., Maaranen, J., Suvanto, M., Pakkanen, T.T. (2014). Solvent-assisted and thermal wrinklings of argon plasma treated polystyrene coatings on silicon substrate, Surf. Coatings Technol., 238, pp. 133-138, DOI: 10.1016/j.surfcoat.2013.10.060.

[40] Chen, C.M., Reed, J.C., Yang, S. (2013). Guided wrinkling in swollen, pre-patterned photoresist thin films with a crosslinking gradient, Soft Matter, 9(46), pp. 11007-11013, DOI: 10.1039/c3sm51881g.

[41] Miquelard-Garnier, G., Croll, A.B., Davis, C.S., Crosby, A.J. (2010). Contact-line mechanics for pattern control, Soft Matter, 6(22), pp. 5789-5794, DOI: 10.1039/c0sm00165a.

[42] Davis, C.S., Crosby, A.J. (2011). Mechanics of wrinkled surface adhesion, Soft Matter, 7(11), pp. 5373-5381, DOI: $10.1039 / \mathrm{c} 1 \mathrm{sm} 05146 \mathrm{f}$.

[43] Chen, Y.C., Crosby, A.J. (2013). Wrinkling of inhomogeneously strained thin polymer films, Soft Matter, 9(1), pp. 4347, DOI: $10.1039 / \mathrm{c} 2 \mathrm{sm} 26822 \mathrm{a}$.

[44] Zhuo, L., Zhang, Y. (2015). The mode-coupling of a stiff film/compliant substrate system in the post-buckling range, Int. J. Solids Struct., 53, pp. 28-37, DOI: 10.1016/j.ijsolstr.2014.10.028.

[45] Zhao, Y., Cao, Y., Feng, X.Q., Ma, K. (2014). Axial compression-induced wrinkles on a core-shell soft cylinder: Theoretical analysis, simulations and experiments, J. Mech. Phys. Solids, 73, pp. 212-227, DOI: $10.1016 /$ j.jmps.2014.09.005.

[46] Mohammadi, H., Müser, M.H. (2010). Friction of wrinkles, Phys. Rev. Lett., 105(22), p. 224301, DOI: $10.1103 /$ PhysRevLett.105.224301.

[47] Vella, D., Ajdari, A., Vaziri, A., Boudaoud, A. (2011). Wrinkling of pressurized elastic shells, Phys. Rev. Lett., 107(17), p. 174301, DOI: 10.1103/PhysRevLett.107.174301.

[48] Bernal, R., Tassius, C., Melo, F., Géminard, J.C. (2011). Elastic response and wrinkling onset of curved elastic membranes subjected to indentation test, Eur. Phys. J. E, 34(2), p. 13, DOI: 10.1140/epje/i2011-11013-0.

[49] Reyes-Martinez, M.A., Ramasubramaniam, A., Briseno, A.L., Crosby, A.J. (2012). The intrinsic mechanical properties of rubrene single crystals, Adv. Mater., 24(41), pp. 5548-5552, DOI: 10.1002/adma.201201749.

[50] Toga, K.B., Huang, J., Cunningham, K., Russell, T.P., Menon, N. (2013). A drop on a floating sheet: Boundary conditions, topography and formation of wrinkles, Soft Matter, 9(34), pp. 8289-8296, DOI: 10.1039/c3sm50736j.

[51] Schroll, R.D., Adda-Bedia, M., Cerda, E., Huang, J., Menon, N., Russell, T.P., Toga, K.B., Vella, D., Davidovitch, B. (2013). Capillary deformations of bendable films, Phys. Rev. Lett., 111(1), p. 014301, DOI: 10.1103/PhysRevLett.111.014301.

[52] Huang, X., Zhao, H.P., Xie, W.H., Hong, W., Feng, X.Q. (2015). Radial wrinkles on film-substrate system induced by local prestretch: A theoretical analysis, Int. J. Solids Struct., 58, pp. 12-19, DOI: 10.1016/j.ijsolstr.2014.12.011.

[53] Lin, P.C., Yang, S. (2007). Spontaneous formation of one-dimensional ripples in transit to highly ordered twodimensional herringbone structures through sequential and unequal biaxial mechanical stretching, Appl. Phys. Lett., 90 , p. 241903, DOI: $10.1063 / 1.2743939$.

[54] Yin, J., Yagüe, J.L., Eggenspieler, D., Gleason, K.K., Boyce, M.C. (2012). Deterministic order in surface microtopologies through sequential wrinkling, Adv. Mater., 24(40), pp. 5441-5446, DOI: 10.1002/adma.201201937.

[55] Yoo, P.J., Suh, K.Y., Park, S.Y., Lee, H.H. (2002). Physical self-assembly of microstructures by anisotropic buckling, Adv. Mater., 14(19), pp. 1383-1387, DOI: 10.1002/1521-4095(20021002)14:19<1383::AID-ADMA1383>3.0.CO;2-D.

[56] Lu, C., Möhwald, H., Fery, A. (2008). Large-scale regioselective formation of well-defined stable wrinkles of multilayered films via embossing, Chem. Mater., 20(22), pp. 7052-7059, DOI: 10.1021/cm8018742.

[57] Yoo, P.J., Lee, H.H. (2008). Complex pattern formation by adhesion-controlled anisotropic wrinkling, Langmuir, 24(13), pp. 6897-6902, DOI: 10.1021/la800126r.

[58] Kundu, S., Davis, C.S., Long, T., Sharma, R., Crosby, A.J. (2011). Adhesion of nonplanar wrinkled surfaces, J. Polym. Sci. Part B Polym. Phys., 49(3), pp. 179-185, DOI: 10.1002/polb.22181.

[59] Yin, J., Lu, C. (2012). Hierarchical surface wrinkles directed by wrinkled templates, Soft Matter, 8(24), pp. 6528-6534, DOI: $10.1039 / \mathrm{c} 2 \mathrm{sm} 25328 \mathrm{c}$.

[60] Huang, J., Davidovitch, B., Santangelo, C.D., Russell, T.P., Menon, N. (2010). Smooth cascade of wrinkles at the edge of a floating elastic film, Phys. Rev. Lett., 105(3), p. 038302, DOI: 10.1103/PhysRevLett.105.038302.

[61] Yu, S.J., Zhang, Y.J., Zhou, H., Chen, M.G., Zhang, X.F., Jiao, Z.W., Si, P.Z. (2013). Spontaneous formation of hierarchical wrinkles in Cr films deposited on silicone oil drops with constrained edges, Phys. Rev. E - Stat. Nonlinear, Soft Matter Phys., 88(4), p. 042401, DOI: 10.1103/PhysRevE.88.042401. 
[62] Vandeparre, H., Léopoldès, J., Poulard, C., Desprez, S., Derue, G., Gay, C., Damman, P. (2007). Slippery or sticky boundary conditions: Control of wrinkling in metal-capped thin polymer films by selective adhesion to substrates, Phys. Rev. Lett., 99(18), p. 188302, DOI: 10.1103/PhysRevLett.99.188302.

[63] Wu, D., Yin, Y., Yang, F., Xie, H. (2014). Mechanism for controlling buckling wrinkles by curved cracks on hard-nanofilm/soft-matter-substrate, Appl. Surf. Sci., 320, pp. 207-212, DOI: 10.1016/j.apsusc.2014.09.072.

[64] Ding, W., Yang, Y., Zhao, Y., Jiang, S., Cao, Y., Lu, C. (2013). Well-defined orthogonal surface wrinkles directed by the wrinkled boundary, Soft Matter, 9(14), pp. 3720-3726, DOI: 10.1039/c2sm27359d.

[65] Ohzono, T., Monobe, H., Shiokawa, K., Fujiwara, M., Shimizu, Y. (2009). Shaping liquid on a micrometre scale using microwrinkles as deformable open channel capillaries, Soft Matter, 5(23), pp.4658-4664, DOI: 10.1039/b912235d.

[66] Yu, C., O’Brien, K., Zhang, Y.H., Yu, H., Jiang, H. (2010). Tunable optical gratings based on buckled nanoscale thin films on transparent elastomeric substrates, Appl. Phys. Lett., 96(4), p. 041111, DOI: 10.1063/1.3298744.

[67] Khang, D.Y., Rogers, J.A., Lee, H.H. (2009). Mechanical buckling: Mechanics, metrology, and stretchable electronics, Adv. Funct. Mater., 19(10), pp. 1526-1536, DOI: 10.1002/adfm.200801065.

[68] Rogers, J.A., Someya, T., Huang, Y. (2010). Materials and mechanics for stretchable electronics, Science, 327(5973), pp. 1603-1607. , DOI: 10.1126 /science.1182383.

[69] Yoo, P.J. (2011). Invited paper: Fabrication of complexly patterned wavy structures using self-organized anisotropic wrinkling, Electron. Mater. Lett., 7(1), pp. 17-23, DOI: 10.1007/s13391-011-0303-8.

[70] Hyun, D.C., Park, M., Park, C., Kim, B., Xia, Y., Hur, J.H., Kim, J.M., Park, J.J., Jeong, U. (2011). Ordered zigzag stripes of polymer gel/metal nanoparticle composites for highly stretchable conductive electrodes, Adv. Mater., 23(26), pp. 2946-2950, DOI: 10.1002/adma.201100639.

[71] Chan, E.P., Smith, E.J., Hayward, R.C., Crosby, A.J. (2008). Surface wrinkles for smart adhesion, Adv. Mater., 20(4), pp. 711-716, DOI: 10.1002/adma.200701530.

[72] Horn, A., Hiltl, S., Fery, A., Böker, A. (2010). Ordering and printing virus arrays: A straightforward way to functionalize surfaces, Small, 6(19), pp. 2122-2215, DOI: 10.1002/smll.201000863.

[73] Stafford, C.M., Harrison, C., Beers, K.L., Karim, A., Amis, E.J., Vanlandingham, M.R., Kim, H.C., Volksen, W., Miller, R.D., Simonyi, E.E. (2004). A buckling-based metrology for measuring the elastic moduli of polymeric thin films, Nat. Mater., 3(8), pp. 545-550, DOI: 10.1038/nmat1175.

[74] Chung, J.Y., Nolte, A.J., Stafford, C.M. (2011). Surface wrinkling: A versatile platform for measuring thin-film properties, Adv. Mater., 23(3), pp. 349-368, DOI: 10.1002/adma.201001759.

[75] Chung, J.Y., Chastek, T.Q., Fasolka, M.J., Ro, H.W., Stafford, C.M. (2009). Quantifying residual stress in nanoscale thin polymer films via surface wrinkling, ACS Nano, 3(4), pp. 844-852, DOI: 10.1021/nn800853y.

[76] Cerda, E., Mahadevan, L. (2003). Geometry and physics of wrinkling, Phys. Rev. Lett., 90(7), p. 074302, DOI: 10.1103/PhysRevLett.90.074302.

[77] Yin, J., Chen, X. (2010). Buckling of anisotropic films on cylindrical substrates: Insights for self-assembly fabrication of 3D helical gears, J. Phys. D. Appl. Phys., 43(11), p. 115402, DOI: 10.1088/0022-3727/43/11/115402.

[78] Pook, L.P., Berto, F., Campagnolo, A. (2017). State of the art of corner point singularities under in-plane and out-ofplane loading, Eng. Fract. Mech., 174, pp. 2-9, DOI: 10.1016/j.engfracmech.2016.10.001.

[79] Chandran, K.S.R. (2018). A novel characterization of fatigue crack growth behavior in metallic materials: The physical relationship between the uncracked section size and the remaining fatigue life, Mater. Sci. Eng. A, 714, pp. 117-123, DOI: 10.1016/j.msea.2017.12.093.

[80] Ravi Chandran, K.S. (2017). A new approach to the mechanics of fatigue crack growth in metals: Correlation of mean stress (stress ratio) effects using the change in net-section strain energy, Acta Mater., 135, pp. 201-214, DOI: 10.1016/j.actamat.2017.06.013.

[81] Pook, L.P. (2016). The linear elastic analysis of cracked bodies, crack paths and some practical crack path examples, Eng. Fract. Mech., 167, pp. 2-19, DOI: 10.1016/j.engfracmech.2016.02.055.

[82] Meneghetti, G., Campagnolo, A., Berto, F. (2016). Assessment of tensile fatigue limit of notches using sharp and coarse linear elastic finite element models, Theor. Appl. Fract. Mech., 84, pp. 106-118, DOI: 10.1016/j.tafmec.2016.06.001.

[83] Pook, L.P., Berto, F., Campagnolo, A., Lazzarin, P. (2014). Coupled fracture mode of a cracked disc under anti-plane loading, Eng. Fract. Mech., 128, pp. 22-36, DOI: 10.1016/j.engfracmech.2014.07.001.

[84] Campagnolo, A., Berto, F., Lazzarin, P. (2015). The effects of different boundary conditions on three-dimensional cracked discs under anti-plane loading, Eur. J. Mech. A/Solids, 50, pp. 76-86, DOI: 10.1016/j.euromechsol.2014.11.001.

[85] Peron, M., Torgersen, J., Berto, F. (2019). Assessment of tensile and fatigue behavior of PEEK specimens in a physiologically relevant environment, 47, pp. 425-436, DOI: 10.3221/IGF-ESIS.47.33. 
[86] Kotousov, A., Lazzarin, P., Berto, F., Pook, L.P. (2013). Three-dimensional stress states at crack tip induced by shear and anti-plane loading, Eng. Fract. Mech., 108, pp. 65-74, DOI: 10.1016/j.engfracmech.2013.04.010.

[87] Berto, F., Lazzarin, P. (2011). Fatigue strength of structural components under multi-axial loading in terms of local energy density averaged on a control volume, Int. J. Fatigue, 33(8), pp. 1055-1065, DOI: 10.1016/j.ijfatigue.2010.11.019.

[88] Berto, F., Campagnolo, A., Elices, M., Lazzarin, P. (2013). A synthesis of Polymethylmethacrylate data from U-notched specimens and V-notches with end holes by means of local energy, Mater. Des., 49, pp. 826-833, DOI: 10.1016/j.matdes.2013.01.074.

[89] Campagnolo, A., Meneghetti, G., Berto, F., Tanaka, K. (2017). Crack initiation life in notched steel bars under torsional fatigue: Synthesis based on the averaged strain energy density approach, Int. J. Fatigue, 100, pp. 563-574, DOI: $10.1016 /$ j.ijfatigue.2016.12.022.

[90] Torabi, A.R., Berto, F., Campagnolo, A., Akbardoost, J. (2017). Averaged strain energy density criterion to predict ductile failure of U-notched Al 6061-T6 plates under mixed mode loading, Theor. Appl. Fract. Mech., 91, pp. 86-93, DOI: $10.1016 /$ j.tafmec.2017.04.010.

[91] Pham, K.H., Ravi-Chandar, K. (2017). The formation and growth of echelon cracks in brittle materials, Int. J. Fract., 206(2), pp. 229-244, DOI: 10.1007/s10704-017-0212-4.

[92] Chen, X., Hutchinson, J.W. (2004). Herringbone buckling patterns of compressed thin films on compliant substrates, J. Appl. Mech., 71(5), pp. 597-603, DOI: 10.1115/1.1756141.

[93] Chen, X., Hutchinson, J.W. (2004). A family of herringbone patterns in thin films, Scr. Mater., 50(6), pp. 797-801, DOI: $10.1016 /$ j.scriptamat.2003.11.035.

[94] Huang, Z.Y., Hong, W., Suo, Z. (2005). Nonlinear analyses of wrinkles in a film bonded to a compliant substrate, J. Mech. Phys. Solids, 53(9), pp. 2101-2118, DOI: 10.1016/j.jmps.2005.03.007.

[95] Bowden, N., Huck, W.T.S., Paul, K.E., Whitesides, G.M. (1999). The controlled formation of ordered, sinusoidal structures by plasma oxidation of an elastomeric polymer, Appl. Phys. Lett., 75(17), pp. 2557-2559, DOI: $10.1063 / 1.125076$.

[96] Singh, K.B., Tirumkudulu, M.S. (2007). Cracking in drying colloidal films, Phys. Rev. Lett., 98(21), p. 218302, DOI: 10.1103/PhysRevLett.98.218302.

[97] Dragnevski, K.I., Routh, A.F., Murray, M.W., Donald, A.M. (2010). Cracking of drying latex films: An ESEM experiment, Langmuir, 26(11), pp. 7747-7751, DOI: 10.1021/la904512q.

[98] Huck, W.T.S., Bowden, N., Onck, P., Pardoen, T., Hutchinson, J.W., Whitesides, G.M. (2000). Ordering of spontaneously formed buckles on planar surfaces, Langmuir, 16(7), pp. 3497-3501, DOI: 10.1021/la9913021.

[99] Yu, C., Jiang, H. (2010). Forming wrinkled stiff films on polymeric substrates at room temperature for stretchable interconnects applications, Thin Solid Films, 519(2), pp. 818-822, DOI: 10.1016/j.tsf.2010.08.106.

[100] Xuan, Y., Guo, X., Cui, Y., Yuan, C., Ge, H., Cui, B., Chen, Y. (2012). Crack-free controlled wrinkling of a bilayer film with a gradient interface, Soft Matter, 8(37), pp. 9603-9609, DOI: 10.1039/c2sm25487e.

[101] Meng, J., Xie, J., Han, X., Lu, C. (2016). Surface wrinkling on polydopamine film, Appl. Surf. Sci., 371, pp. 96-101, DOI: 10.1016/j.apsusc.2016.02.239.

[102] Piñeirua, M., Tanaka, N., Roman, B., Bico, J. (2013). Capillary buckling of a floating annulus, Soft Matter, 9(46), pp. 10985-10992, DOI: 10.1039/c3sm51825f.

[103] Yu, S.J. (2014). Wrinkle patterns of Fe films deposited on micro-scale silicone oil droplets, Thin Solid Films, 558, pp. 247-251, DOI: $10.1016 /$ j.tsf.2014.03.037.

[104] Deng, S., Berry, V. (2016). Increased hierarchical wrinklons on stiff metal thin film on a liquid meniscus, ACS Appl. Mater. Interfaces, 8(37), pp. 24956-24961, DOI: 10.1021/acsami.6b08027.

[105] Yin, J., Chen, X. (2010). Elastic buckling of gradient thin films on compliant substrates, Philos. Mag. Lett., 90(6), pp. 423-433, DOI: 10.1080/09500831003745258.

[106] Ni, Y., Yang, D., He, L. (2012). Spontaneous wrinkle branching by gradient stiffness, Phys. Rev. E - Stat. Nonlinear, Soft Matter Phys., 86(3), p. 031604, DOI: 10.1103/PhysRevE.86.031604.

[107] Yin, J., Chen, X., Sheinman, I. (2009). Anisotropic buckling patterns in spheroidal film/substrate systems and their implications in some natural and biological systems, J. Mech. Phys. Solids, 57(9), pp. 1470-1484, DOI: $10.1016 /$ j.jmps.2009.06.002.

[108] Dervaux, J., Couder, Y., Guedeau-Boudeville, M.A., Ben Amar, M. (2011). Shape transition in artificial tumors: From smooth buckles to singular creases, Phys. Rev. Lett., 107(1), p. 018103, DOI: 10.1103/PhysRevLett.107.018103.

[109] Ben Amar, M., Wu, M. (2014). Patterns in biofilms: From contour undulations to fold focussing, EPL, 108(3), p. 38003, DOI: 10.1209/0295-5075/108/38003. 
[110] Espeso, D.R., Carpio, A., Einarsson, B. (2015). Differential growth of wrinkled biofilms, Phys. Rev. E - Stat. Nonlinear, Soft Matter Phys., 91(2), p. 022710, DOI: 10.1103/PhysRevE.91.022710.

[111] Tallinen, T., Chung, J.Y., Rousseau, F., Girard, N., Lefèvre, J., Mahadevan, L. (2016). On the growth and form of cortical convolutions, Nat. Phys., 12 (6), pp. 588-593, DOI: 10.1038/nphys3632.

\section{NOMENCLATURE}

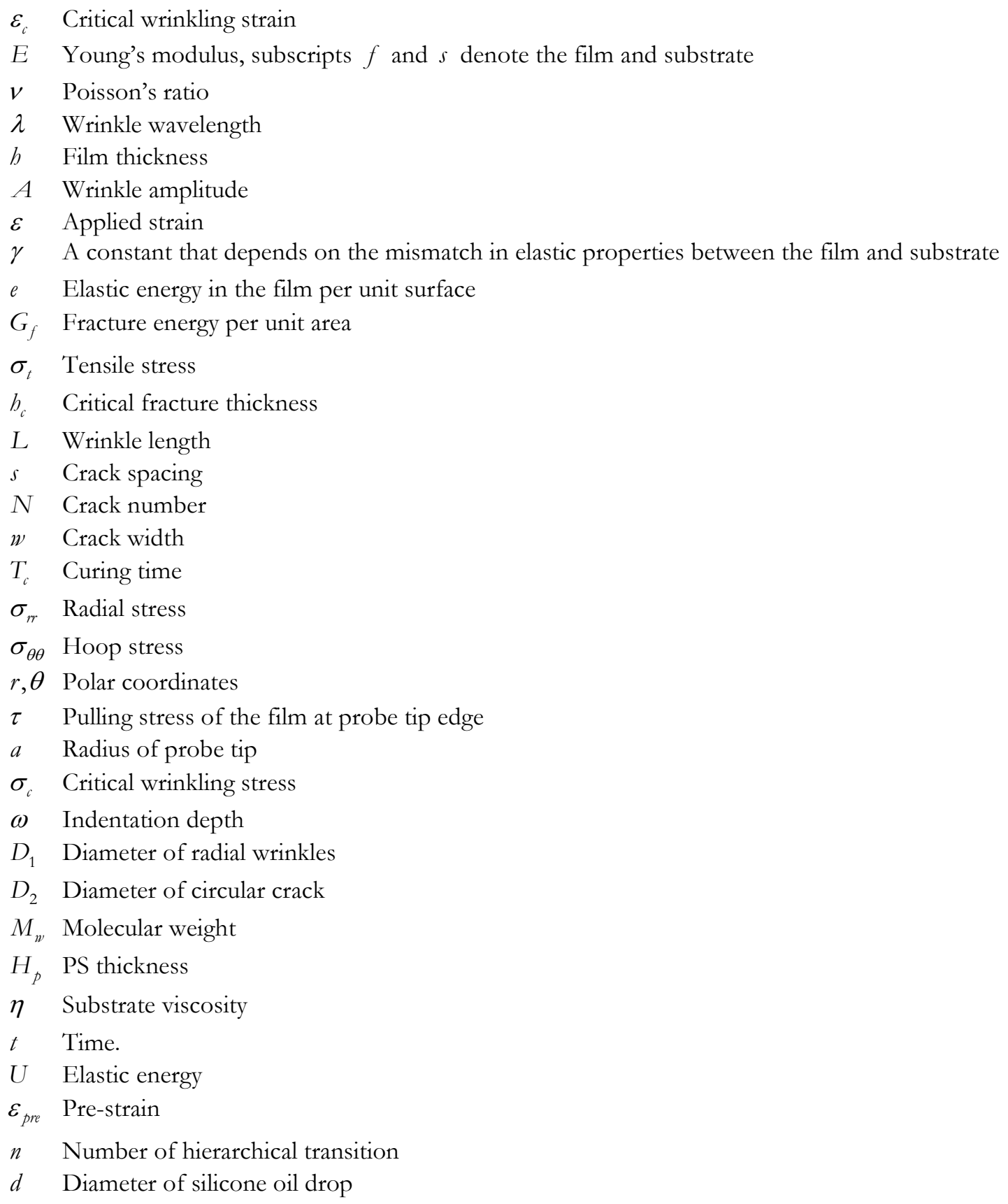

